\title{
Role of mechanical morphogenesis in the development and evolution of the neocortex
}

\author{
Katja Heuer ${ }^{1,2}$ and Roberto Toro ${ }^{1,3}$ \\ ${ }^{1}$ Groupe de Neuroanatomie Appliquée et Théorique, Département de Neuroscience, Institut Pasteur, Paris, France \\ ${ }^{2}$ Max Planck Institute for Human Cognitive and Brain Sciences, Leipzig, Germany \\ ${ }^{3} \mathrm{CRI}$, Université Paris Descartes, Inserm U 1001, Paris, France
}

\begin{abstract}
During the short period of brain development, nature is able to build the only system we know capable of producing cognition, language, creativity, and consciousness. The neocortex - the outermost layer of the mammalian cerebrum - appears to be the biological substrate of these abilities. Its development requires not only the precise placement and wiring of billions of cells, but also the implementation of mechanisms to ensure a viable cognition despite sometimes dramatic perturbations. Today, this remarkably complex organisation is thought to be genetically encoded, and further refined by activity-dependent processes. We propose that mechanical morphogenesis - the capacity of homogeneously growing elastic tissue to produce complex shapes - can also play an important role. Out of homogeneous growth, mechanical morphogenesis can induce the segregation of the neocortex into mechanical and geometric modules - the neocortical folds. Through the feedback of physical forces on developing tissue, these modules can influence the differentiation and wiring of the neocortex, having a causal role on neocortical development, and providing adaptable and robust units for its evolution.
\end{abstract}

\section{Introduction}

How is the remarkable complexity of the mammalian neocortex produced? From mice to whales, mammalian neocortices are a relatively thin layer of neuronal bodies, differentiated into a series of interconnected cytoarchitectonic fields. Neocortical surface area is extremely variable across species, from about $1.5 \mathrm{~cm}^{2}$ in mice, $1500 \mathrm{~cm}^{2}$ in humans, to $3,000 \mathrm{~cm}^{2}$ in pilot whales (Mota and Herculano-Houzel 2015). Its thickness, by contrast, only varies from about $1 \mathrm{~mm}$ in mice to $2.5 \mathrm{~mm}$ in humans. In the direction of its thickness, the neocortex can be divided into 6 main layers. The connections among those layers tend to follow a canonical circuit: neurones in layer 4 receive connections from subcortical structures, project to those in the superficial layers which then send projections to other neocortical areas and to the deep layers below layer 4. Neurones in deep layers mostly send projections to regions outside the neocortex (Felleman and Van Essen 1991, Douglas and Martin 2004). Variations in layer thickness, presence of sub-layers, neuronal morphology, intracortical myelination, but also connectivity, chemical receptor distribution and gene 
expression, allow us to distinguish different neocortical regions - the cytoarchitectonic areas. Some of these areas exhibit well described relationships with sensory and motor functions. For example, the primary visual cortex or V1, located in the posterior part of the brain, contains a complete "retinotopic" map of the visual field: neighbouring locations of the eye's retina occupy neighbouring locations in V1. Furthermore, different cell types in the retina project to different sublayers of V1's layer 4 , and these connections are segregated into ocular dominance bands preferentially related to the left or the right eye. $\mathrm{V} 1$ has connections with various other visual areas that have been related to several other aspects of visual perception, as well as with associative areas combining multiple sensory modalities (Felleman and Van Essen 1991).

An interesting observation, dating from the first brain cartography studies at the beginning of the $20^{\text {th }}$ century, is the puzzling relationship between the cytoarchitectonic organisation of the neocortex, its connections and functions, with the geometry of its sulci and gyri - the valleys and hills of the cerebrum (Brodmann 1909). Many examples of this relationship exist. In humans and other primates, a deep fold called the central sulcus separates the somatosensory cortex from the motor cortex. In the primary visual cortex, another deep fold, the calcarine sulcus, separates the superior and inferior visual hemifields. Experiments in animals such as ferrets or racoons show that sulci in the somatosensory cortex often appear silent in electrophysiological recordings, whereas gyri readily respond to sensory stimulation of different parts of the body (Welker and Campos 1963, Leclerc et al 1993, Rice et al 1993). In addition to these cytoarchitectonic and functional differences, brain connectivity also shows a relationship with sulco-gyral anatomy. For example, a ubiquitous type of short association fibres, the $U$-fibres, connect regions in neighbouring gyri: axonal fibres are perpendicular to the neocortical surface in the top of gyri, but tangential in the bottom of sulci (Catani et al 2012, Thiebaut de Schotten et al 2012, Cottaar et al 2018).

\section{Genetic and activity-dependent processes}

There is agreement today that the development of neocortical organisation follows a genetically encoded template, a protomap, refined by activity-dependent processes (Rakic 1988, Krubitzer 2007, Rakic 2009, O'Leary et al 2013, Renier et al 2017). A series of experiments in mice suggest that the general layout of the neocortex is deployed through the establishment of molecular gradients from a reduced number of patterning centres. Through these gradients, each neocortical location would be characterised by a unique combination of molecules, which would then trigger the development of a specific type of cortex (O'Leary et al 2013). For example, the experimental alteration of the gradient of one of these molecules - the transcription factor EMX2 - produces the displacement of the mouse barrel cortex (the neocortical region that responds to the stimulation of individual whiskers), and even its duplication (Fukuchi-Shimogori and Grove 2003, Hamasaki et al 2004). The global coordinate system produced by molecular gradients would then allow the local specification of cytoarchitectonic areas and their connections: each region would be genetically programmed to attract specific types of connections, and produce fibres aiming to connect with specific targets. Further refinement of this layout would then be achieved through activity-dependent processes, such as visual stimulation. For example, it has been observed that 
ocular dominance bands fail to develop if visual activity is prevented (Katz and Shatz 1996, Crowley and Katz 2002). More impressively, experiments in mice, hamsters and ferrets where visual connections were made to innervate the auditory cortex showed that visual activity was able to induce a cytoarchitecture in the auditory cortex that resembles strikingly that of a normal visual cortex (Sur et al 1988, Sharma et al 2000, Newton et al 2004).

As we said, most support for the idea of a genetic encoding of neocortical organisation comes from experiments in mice, and we need to keep in mind that mice are at the lowest extreme of mammalian brain size, have a comparatively small number of cytoarchitectonic fields, little cortico-cortical connectivity, and no neocortical folding. It would be nevertheless easy to extend the principle to encompass cases of more complex neocortical organisations. We could imagine that mammals with larger neocortices also evolved a more complex genetic decoding mechanism from global molecular coordinates into cytoarchitectonic regions and cortico-cortical connections.

We could also easily extend this idea to explain the correspondence of cytoarchitectonic and connective organisation with sulci and gyri: the same genetic program that determines the specification of different areas and their connections, could also encode the pattern of brain folds. Several hypotheses about the way in which this could be implemented have been indeed proposed through the years. For example, it has been proposed that the tension along axons connecting different regions could make the neocortex fold, explaining the relationship between folding and connectivity (Van Essen 1997). It has also been proposed that the genetic protomap could encode the differential growth of some areas (Ronan and Fletcher 2015), either by driving gyral regions to grow more (Welker 1990, De Juan Romero et al 2014) or by preventing sulci from moving (Smart and McSherry 1986).

However, recent theoretical and experimental results suggest that brain folding is an autonomous process, and that neocortical growth on top of the elastic white matter substrate should be sufficient to produce cortical folding. If this is indeed the case, we need a different explanation for the relationship between cytoarchitectonic and connective organisation with the sulcal/gyral anatomy. The potential implications are very important because brain folding, which was supposed to be an effect, could turn out indeed to be a cause.

\section{Mechanical morphogenesis}

Brain tissue, grey and white matter, has mechanical properties similar to those of jelly: it is elastic, and largely incompressible (Tallinen et al 2014). During development, the neocortex develops from the cumulation of successive waves of neurones migrating from the inner core of the cerebrum the ventricular and outer subventricular zones (Reillo et al 2011). After the end of neuronal migration the cerebral hemispheres are still smooth (unfolded) and neurones are densely packed. The growth of intracortical connections, dendritic trees and glial proliferation then produces a rapid neocortical expansion. In humans, the neocortex is still unfolded at 20 weeks of gestation, and expands from $\sim 80 \mathrm{~cm}^{2}$ at that time to $\sim 600 \mathrm{~cm}^{2}$ at birth, when it is profusely folded - a 7.5 -fold 
increase in surface area (Dubois et al 2007, Garcia et al 2018). During the equivalent period in ferrets, from postnatal day 0 until 1 month of age, the neocortex expands 10 -fold, from $\sim 3 \mathrm{~cm}^{2}$ to $\sim 30 \mathrm{~cm}^{2}$ (Knutsen et al 2013). Results from soft matter physics show that folds, wrinkles, ridges and creases, are the expected outcomes in such systems (Goriely 2017). An elastic layer such as the neocortex, growing on top of an elastic substrate such as the white matter, will first grow without folding; but if growth continues, the cost of bending the neocortex will become energetically less than that required to keep expanding the white matter, leading to folding. Several models of brain folding have been built on this principle (Toro and Burnod 2005, Bayly et al 2013, Tallinen et al 2014, Budday et al 2014). These models are able to reproduce the dynamics of brain folding, their shape, the degree of folding of brains as a function of their size, and even the differences in thickness characteristic of sulcal and gyral regions. They show that even some aspects of the pattern of neocortical folding - the locations where they form - could be explained by mechanical and geometrical properties (Toro and Burnod 2005, Toro 2012, Tallinen et al 2014, 2016).

The mechanical formation of folding should induce, in addition to the large geometric deformation of the tissue, a complex pattern of stress gradients at the scale of the complete organ (Nelson 2016, Goriely 2017, Foubet et al 2018). Developing tissue is very sensitive to its mechanical environment (Franze, 2013). The feedback of stress gradients onto biological tissue could trigger a variety of responses. In vitro experiments show that mechanical forces can modulate cell proliferation and programmed cell death (Saha et al 2008, Montel et al 2012). Differences in substrate stiffness can determine whether neuronal progenitors produce glial cells or neurones (Saha et al 2008). Softer substrates have been observed to promote neuronal dendritic branching (Flanagan et al 2002). Finally, axons tend to follow the stiffer directions when cultured on a mechanically anisotropic substrate (Saez et al 2007).

Without requiring a genetic prescription of neocortical organisation, the formation of brain folding could induce a stable and complex pattern of regional cellular changes and neuronal connections. Together with genetic and activity-dependent processes, mechanical morphogenesis, and in particular brain folding, could play an important role in the development and evolution of neocortical organisation.

\section{Testing mechanical morphogenesis}

While there is a variety of methodologies for probing the causal effect of activity-dependent and genetic processes, no equivalent method exists today for testing mechanical morphogenetic processes in vivo. Perturbation methodologies such as sensory deprivation, enucleation of the eyes, activity blockade, optogenetics, gene knockouts, knockins, localised electroporation or CRISPR gene-editing, allow researchers to study the effects of neuronal activity and genetics on brain development. Moreover, through the years, the relevant methods have also been developed to control for unwanted side effects of these interventions such as inflammation or toxicity. Probing the role of mechanical morphogenesis on the development of folding and neocortical organisation 
requires similar methodologies to introduce a perturbation while controlling for undesired side effects.

A few examples of mechanical perturbations of brain folding exist in the literature. Barron (1950) studied the role of the constraint imposed by the cranium on brain folding in developing sheep. As many herbivores, sheep have a rather thin and profusely folded neocortex. Barron surgically extracted in utero up to one complete hemisphere. If the brain folds because of the limited intracranial space, the remaining hemisphere should be able to grow unconstrained, and thus unfolded. On the contrary, he observed at birth a degree and a pattern of folding of the remaining hemisphere very similar to those of normal sheep. Welker (1990) aimed at testing the possibility of altering an already established folding pattern in cats and racoons. Removing a large portion of the neocortex in cats, he was able to change the anterior-posterior course of many folds into an orthogonal inferior-superior direction. In racoons, he removed most of the white matter inside the temporal lobe. Released from the white matter, the neocortex lost its folds (Welker 1990).

The experiments of Barron (1950) and Welker (1990) were extremely invasive, and the knowledge gained from mathematical modelling should help us design less invasive, more reproducible methodologies. If brain folding results from a mechanical instability, then the neocortex should be at a maximally unstable configuration by the time immediately preceding the formation of the first folds. Furthermore, mechanical models can be used to predict the most likely orientations in which folds will develop. These orientations correspond to the principal directions of curvature of the surface - the two orthogonal directions at each point of a surface where mean curvature reaches its minimum and its maximum (Toro 2012, Tallinen et al 2014, 2016). At this time, even a small perturbation introduced at the right location should suffice to trigger the development of a fold. Ferrets have become important animal models for the study of brain folding, in particular, because brain folding starts in ferrets during the first few days after birth (in humans folding starts during the last trimester of gestation). A mechanical perturbation could be introduced surgically in a ferret kit, for example, by implanting a soft rod between the cranium and the neocortical surface. However, a completely non-invasive perturbation could be performed, for example, using ultrasonic technology. Acoustic radiation force (Gennisson et al 2013) can produce forces strong enough to levitate and manipulate small objects without contact, and can be maintained for many hours without damaging the tissues. The skull of ferret kits is still thin, and ultrasonic stimulation should not require surgical interventions. Ultrasonic tomography could also be used to monitor in vivo the structure, vascularisation and mechanical properties of the tissue. Ultrasonic recordings could be carried out longitudinally, to monitor the evolution of the experiment without further intervention.

A successful mechanical perturbation should allow us to observe the effects of geometric changes and residual stress gradients on brain organisation, and potentially even on behaviour. If the orientation of brain folding follows indeed one of the two principal directions of curvature, it should be possible to force the selection of the second one: regions folded along the anterior-posterior orientation should fold in the superior-inferior direction (as hinted by the experiments of Welker (1990)). In ferrets, various sulci limit cytoarchitectonic and functional fields. If mechanical morphogenesis plays a role in this organisation, these boundaries should transform along with the 
sulci. Several neighbouring gyri in ferrets are connected through U-shaped short fibres. Altering the orientation of brain folding should also alter this pattern, leading to the connection of regions that have never been connected in normal animals. Subcortical structures such as thalamic nuclei are preferentially connected to specific gyri, in particular, those related to different sensory-motor modalities. An altered folding pattern should also change the cortico-subcortical connectivity, with measurable behavioural consequences. In the case of rewired mice and ferrets, for example, where visual activity was made to innervate auditory cortex, some of the characteristics of auditory conditioning were transferred to visual conditioning (Melchner et al 2000, Newton et al 2004).

\section{Discussion and conclusion}

Mechanical morphogenesis could play an important role on the development and evolution of neocortical organisation. Instead of a detailed genetic prescription of neocortical areas and their connectivity, the growth of the neocortex over the white matter should suffice to trigger the formation of residual stress gradients at the scale of the complete cerebrum, as well as localised modules with prototypic geometry and mechanical properties - the neocortical folds. This could spark a series of responses in the developing tissue, transforming mechanical modules into cytoarchitectonic, connective and functional modules. For the moment we can only speculate about the breadth of the effects that mechanical morphogenesis can have on the developing neocortex in vivo, based on results largely obtained in vitro. The design of appropriate in vivo mechanical perturbation methodologies is a major challenge for further research.

The main determinant of the wavelength of folding is neocortical thickness, which is remarkably stable across mammals. As far as total cortical thickness stays unchanged, brain folds should conserve their geometry and the residual stress patterns they produce. This provides a stable, resilient, scaffold for the developmental processes influenced by folding, as well as a reproducible unit over which evolution can operate. Mechanical models of folding predict that wavelength $\lambda$ should be directly proportional to neocortical thickness $t$ (Bayly et al 2013, Tallinen et al 2014). If the elasticity of the grey and the white matter were comparable, then $\lambda \sim 4.4$ t (Tallinen et al 2014). We have recently studied the variation of fold wavelength in 33 primate species (Heuer et al 2018). From vervet monkeys to humans, the wavelength was of $12 \pm 2.4$ (in close agreement with the mechanical model prediction) despite an 18-fold variation in brain volume.

Evolution requires structures able at the same time to change and adapt, but also sufficiently stable to conserve their fitness (Conrad 1990). Such "evolvable" structures should be stable for pathological variation, but unstable for physiological variation. Conrad (1990) suggests that this can be achieved through compartmentalisation - the creation of relatively independent modules, and component redundancy - the development of repeated modules with similar characteristics. These structures should be produced through multiple independent evolutionary paths so as to ensure their robustness (Lewontin 1978, Conrad 1990). The modules created by brain folding fulfill the characteristics of an evolvable structure: they provide a mechanism to create a series of independent modules with similar properties. Their formation can be controlled by a single, strongly 
polygenic parametre, neocortical growth. Variations in neocortical growth in humans have been shown to be extremely polygenic, resulting from the additive combination of large numbers of small effects (Toro et al 2014, Chen et al 2015, Zhao et al 2018). Whereas the differences in fold wavelength are extremely small, there is a 2-fold inter-individual variation in total cortical surface area and hence in the degree of folding (Toro et al 2008). Modules regulated by brain folding should be then both resilient to a large number of genetic variations affecting neocortical growth, but able to produce an increased number of redundant modules across species, and even within species, without requiring major changes in genetic architecture.

Within this framework, the role of genetic processes would not be to completely encode neocortical organisation up to the level of the individual fold, but to select, regulate and consolidate the variety of developmental patterns that mechanical morphogenesis makes available. Instead of a precise prescription of neocortical regions, a global regulation of neocortical growth should lead to the formation of a series of complex cytoarchitectonic, connective and functional modules. Through activity-dependent processes, these patterns of differentiation and connectivity could be refined to match functional and environmental requirements. In addition to global neocortical growth, a finer control of mechanical morphogenetic processes could be exerted by producing gradual changes in growth rate and timing, or by controlling the developmental processes sculpting the shape of the telencephalon before the onset of neocortical folding. Mechanical morphogenesis could then generate robust and reproducible mechanical modules, providing a physically buffered substrate for development and evolution of the neocortex.

\section{References}

1. Bayly, P. V., Okamoto, R. J., Xu, G., Shi, Y., \& Taber, L. A. (2013). A cortical folding model incorporating stress-dependent growth explains gyral wavelengths and stress patterns in the developing brain. Physical Biology, 10(1), 16005.

https://doi.org/10.1088/1478-3975/10/1/016005

2. Barron, D. H. (1950). An experimental analysis of some factors involved in the development of the fissure pattern of the cerebral cortex. Journal of Experimental Zoology, 113(3), 553-581. https://doi.org/10.1002/jez.1401130304

3. Budday, S., Raybaud, C., \& Kuhl, E. (2014). A mechanical model predicts morphological abnormalities in the developing human brain. Scientific Reports, 4(1).

https://doi.org/10.1038/srep05644

4. Brodmann, K. (1909). Vergleichende Lokalisationslehre der Großhirnrinde in ihren Prinzipien dargestellt auf Grund des Zellenbaues. Leipzig: Johann Ambrosius Barth Verlag.

5. Catani, M., Dell'Acqua, F., Vergani, F., Malik, F., Hodge, H., Roy, P., ... Thiebaut de Schotten, M. (2012). Short frontal lobe connections of the human brain. Cortex, 48(2), 273-291. https://doi.org/10.1016/i.cortex.2011.12.001

6. Chen, C.-H., Peng, Q., Schork, A. J., Lo, M.-T., ... Fan, C.-C. (2015). Large-scale genomics unveil polygenic architecture of human cortical surface area. Nature Communications, 6(1). https://doi.org/10.1038/ncomms8549 
7. Cottaar, M., Bastiani, M., Chen, C., Dikranian, K., Van Essen, D., Behrens, T. E., ... Jbabdi, S. (2018). A gyral coordinate system predictive of fibre orientations. Neurolmage, 176, 417-430. https://doi.org/10.1016/..neuroimage.2018.04.040

8. Conrad, M. (1990). The geometry of evolution. Biosystems, 24(1), 61-81. https://doi.org/10.1016/0303-2647(90)90030-5

9. Crowley, J. C., \& Katz, L. C. (2002). Ocular dominance development revisited. Current Opinion in Neurobiology, 12(1), 104-109. https://doi.org/10.1016/s0959-4388(02)00297-0

10. de Juan Romero, C., Bruder, C., Tomasello, U., Sanz-Anquela, J. M., \& Borrell, V. (2015). Discrete domains of gene expression in germinal layers distinguish the development of gyrencephaly. The EMBO Journal, 34(14), 1859-1874. https://doi.org/10.15252/embj.201591176

11. Douglas, R. J., \& Martin, K. A. C. (2004). Neuronal circuits of the neocortex. Annual Review of Neuroscience, 27(1), 419-451. https://doi.org/10.1146/annurev.neuro.27.070203.144152

12. Dubois, J., Benders, M., Cachia, A., Lazeyras, F., Ha-Vinh Leuchter, R., Sizonenko, S. V., ... Huppi, P. S. (2007). Mapping the Early Cortical Folding Process in the Preterm Newborn Brain. Cerebral Cortex, 18(6), 1444-1454. https://doi.org/10.1093/cercor/bhm180

13. Felleman, D. J., \& Van Essen, D. C. (1991). Distributed Hierarchical Processing in the Primate Cerebral Cortex. Cerebral Cortex, 1(1), 1-47. https://doi.org/10.1093/cercor/1.1.1

14. Flanagan L. A., Ju Y. E., Marg B., Osterfield M., Janmey P. A. (2002). Neurite branching on deformable substrates. NeuroReport, 13(18), 2411-2415. https://dx.doi.org/10.1097/01.wnr.0000048003.96487.97

15. Foubet, O., Trejo, M., \& Toro, R. (2018). Mechanical morphogenesis and the development of neocortical organisation. Cortex. https://doi.org/10.1016/j.cortex.2018.03.005

16. Franze, K. (2013). The mechanical control of nervous system development. Development, 140(15), 3069-3077. https://doi.org/10.1242/dev.079145

17. Fukuchi-Shimogori, T., \& Grove, E. A. (2003). Emx2 patterns the neocortex by regulating FGF positional signaling. Nature Neuroscience, 6(8), 825-831. https://doi.org/10.1038/nn1093

18. Garcia, K. E., Robinson, E. C., Alexopoulos, D., Dierker, D. L., Glasser, M. F., Coalson, T. S., ... Bayly, P. V. (2018). Dynamic patterns of cortical expansion during folding of the preterm human brain. Proceedings of the National Academy of Sciences, 115(12), 3156-3161. https://doi.org/10.1073/pnas.1715451115

19. Gennisson, J.-L., Deffieux, T., Fink, M., \& Tanter, M. (2013). Ultrasound elastography: Principles and techniques. Diagnostic and Interventional Imaging, 94(5), 487-495. https://doi.org/10.1016/i.diii.2013.01.022

20. Goriely, A. (2017). The Mathematics and Mechanics of Biological Growth. Interdisciplinary Applied Mathematics. Springer New York. https://doi.org/10.1007/978-0-387-87710-5

21. Hamasaki, T., Leingärtner, A., Ringstedt, T., \& O'Leary, D. D. M. (2004). EMX2 Regulates Sizes and Positioning of the Primary Sensory and Motor Areas in Neocortex by Direct Specification of Cortical Progenitors. Neuron, 43(3), 359-372. https://doi.org/10.1016/i.neuron.2004.07.016 
22. Heuer et al (2018) Heuer, K., Gulban, O. F., Bazin, P.-L., Osoianu, A., Valabregue, R., Santin, M., ... Toro, R. (2018, July 30). Evolution of neocortical folding: A phylogenetic comparative analysis of MRI from 33 primate species. biorXiv.

https://doi.org/10.1101/379750

23. Katz, L. C., \& Shatz, C. J. (1996). Synaptic Activity and the Construction of Cortical Circuits. Science, 274(5290), 1133-1138. https://doi.org/10.1126/science.274.5290.1133

24. Knutsen, A. K., Kroenke, C. D., Chang, Y. V., Taber, L. A., \& Bayly, P. V. (2012). Spatial and Temporal Variations of Cortical Growth during Gyrogenesis in the Developing Ferret Brain.

Cerebral Cortex, 23(2), 488-498. https://doi.org/10.1093/cercor/bhs042

25. Krubitzer, L. (2007). The Magnificent Compromise: Cortical Field Evolution in Mammals. Neuron, 56(2), 201-208. https://doi.org/10.1016/j.neuron.2007.10.002

26. Leclerc, S. S., Rice, F. L., Dykes, R. W., Pourmoghadam, K., \& Gomez, C. M. (1993). Electrophysiological Examination of the Representation of the Face in the Suprasylvian Gyrus of the Ferret: A Correlative Study with Cytoarchitecture. Somatosensory \& Motor Research, 10(2), 133-159. https://doi.org/10.3109/08990229309028829

27. Lewontin, R. C. (1978). Adaptation. Scientific American, 239(3), 212-230. https://doi.org/10.1038/scientificamerican0978-212

28. von Melchner, L., Pallas, S. L., \& Sur, M. (2000). Visual behaviour mediated by retinal projections directed to the auditory pathway. Nature, 404(6780), 871-876. https://doi.org/10.1038/35009102

29. Mota, B., \& Herculano-Houzel, S. (2015). Cortical folding scales universally with surface area and thickness, not number of neurons. Science, 349(6243), 74-77.

https://doi.org/10.1126/science.aaa9101

30. Nelson, C. M. (2016). On Buckling Morphogenesis. Journal of Biomechanical Engineering, 138(2), 21005. https://doi.org/10.1115/1.4032128

31. Newton, J. R., Ellsworth, C., Miyakawa, T., Tonegawa, S., \& Sur, M. (2004). Acceleration of visually cued conditioned fear through the auditory pathway. Nature Neuroscience, 7(9), 968-973. https://doi.org/10.1038/nn1306

32. O'Leary, D.D.M., Stocker, A.M., Zembrzycki, A., and Rakic, J.L.R.R. (2013). Chapter 4 Area Patterning of the Mammalian Cortex. In Patterning and cell type specification in the developing CNS and PNS. Oxford: Academic Press, pp. 61-85.

33. Rakic, P. (1988). Specification of cerebral cortical areas. Science, 241(4862), 170-176. https://doi.org/10.1126/science.3291116

34. Rakic, P. (2009). Evolution of the neocortex: a perspective from developmental biology. Nature Reviews Neuroscience, 10(10), 724-735. https://doi.org/10.1038/nrn2719

35. Reillo, I., de Juan Romero, C., García-Cabezas, M. Á., \& Borrell, V. (2010). A Role for Intermediate Radial Glia in the Tangential Expansion of the Mammalian Cerebral Cortex. Cerebral Cortex, 21(7), 1674-1694. https://doi.org/10.1093/cercor/bhq238

36. Renier, N., Dominici, C., Erzurumlu, R. S., Kratochwil, C. F., Rijli, F. M., Gaspar, P., \& Chédotal, A. (2017). A mutant with bilateral whisker to barrel inputs unveils somatosensory mapping rules in the cerebral cortex. eLife, 6. https://doi.org/10.7554/elife.23494

37. Rice, F. L., Gomez, C. M., Leclerc, S. S., Dykes, R. W., Moon, J. S., \& Pourmoghadam, K. (1993). Cytoarchitecture of the Ferret Suprasylvian Gyrus Correlated with Areas Containing 
Multiunit Responses Elicited by Stimulation of the Face. Somatosensory \& Motor Research, 10(2), 161-188. https://doi.org/10.3109/08990229309028830

38. Ronan, L., \& Fletcher, P. C. (2014). From genes to folds: a review of cortical gyrification theory. Brain Structure and Function, 220(5), 2475-2483.

https://doi.org/10.1007/s00429-014-0961-z

39. Saha, K., Keung, A. J., Irwin, E. F., Li, Y., Little, L., Schaffer, D. V., \& Healy, K. E. (2008). Substrate Modulus Directs Neural Stem Cell Behavior. Biophysical Journal, 95(9), 4426-4438. https://doi.org/10.1529/biophysj.108.132217

40. Saez, A., Ghibaudo, M., Buguin, A., Silberzan, P., \& Ladoux, B. (2007). Rigidity-driven growth and migration of epithelial cells on microstructured anisotropic substrates. Proceedings of the National Academy of Sciences, 104(20), 8281-8286. https://doi.org/10.1073/pnas.0702259104

41. Sharma, J., Angelucci, A., \& Sur, M. (2000). Induction of visual orientation modules in auditory cortex. Nature, 404(6780), 841-847. https://doi.org/10.1038/35009043

42. Smart, I. H., \& McSherry, G. M. (1986). Gyrus formation in the cerebral cortex of the ferret. II. Description of the internal histological changes. Journal of anatomy, 147, 27.

43. Sur, M., Garraghty, P., \& Roe, A. (1988). Experimentally induced visual projections into auditory thalamus and cortex. Science, 242(4884), 1437-1441.

https://doi.org/10.1126/science.2462279

44. Tallinen, T., Chung, J. Y., Biggins, J. S., \& Mahadevan, L. (2014). Gyrification from constrained cortical expansion. Proceedings of the National Academy of Sciences, 111(35), 12667-12672. https://doi.org/10.1073/pnas.1406015111

45. Tallinen, T., Chung, J. Y., Rousseau, F., Girard, N., Lefèvre, J., \& Mahadevan, L. (2016). On the growth and form of cortical convolutions. Nature Physics, 12(6), 588-593. https://doi.org/10.1038/nphys3632

46. Thiebaut de Schotten, M., Dell'Acqua, F., Valabregue, R., \& Catani, M. (2012). Monkey to human comparative anatomy of the frontal lobe association tracts. Cortex, 48(1), 82-96. https://doi.org/10.1016/..cortex.2011.10.001

47. Toro, R., \& Burnod, Y. (2005). A Morphogenetic Model for the Development of Cortical Convolutions. Cerebral Cortex, 15(12), 1900-1913. https://doi.org/10.1093/cercor/bhi068

48. Toro, R., Perron, M., Pike, B., Richer, L., Veillette, S., Pausova, Z., \& Paus, T. (2008). Brain Size and Folding of the Human Cerebral Cortex. Cerebral Cortex, 18(10), 2352-2357. https://doi.org/10.1093/cercor/bhm261

49. Toro, R. (2012). On the Possible Shapes of the Brain. Evolutionary Biology, 39(4), 600-612. https://doi.org/10.1007/s11692-012-9201-8

50. Toro, R., Poline, J.-B., Huguet, G., Loth, E., Frouin, V., Banaschewski, T., ... Bourgeron, T. (2014). Genomic architecture of human neuroanatomical diversity. Molecular Psychiatry, 20(8), 1011-1016. https://doi.org/10.1038/mp.2014.99

51. Van Essen, D. C. (1997). A tension-based theory of morphogenesis and compact wiring in the central nervous system. Nature, 385(6614), 313-318.

https://doi.org/10.1038/385313a0

52. Welker, W. (1990). Why Does Cerebral Cortex Fissure and Fold? In Cerebral Cortex (pp. 3-136). Springer US. https://doi.org/10.1007/978-1-4615-3824-0 1 
53. Zhao, B., Ibrahim, J. G., Li, Y., Li, T., Wang, Y., Shan, Y., ... Zhu, H. (2018). Heritability of Regional Brain Volumes in Large-Scale Neuroimaging and Genetic Studies. Cerebral Cortex. https://doi.org/10.1093/cercor/bhy157 